\title{
L’apprivoisement du pouvoir : l'apprentissage épiscopal de Pierre Denaut et de Joseph-Octave Plessis
}

\section{James H. Lambert}

Volume 51, 1984

URI : https://id.erudit.org/iderudit/1007448ar

DOI : https://doi.org/10.7202/1007448ar

Aller au sommaire du numéro

Éditeur(s)

Les Éditions Historia Ecclesiæ Catholicæ Canadensis Inc.

ISSN

0318-6172 (imprimé)

1927-7067 (numérique)

Découvrir la revue

Citer cet article

Lambert, J. H. (1984). L’apprivoisement du pouvoir : l'apprentissage épiscopal de Pierre Denaut et de Joseph-Octave Plessis. Sessions d'étude - Société canadienne d'histoire de l'Église catholique, 51, 9-17.

https://doi.org/10.7202/1007448ar
Résumé de l'article

Le caractère et la carrière épiscopale respectifs de Pierre Denaut, évêque de Québec de 1797 à 1806, et de son successeur Joseph-Octave Plessis, expliquent en partie leurs façons différentes d'aborder les problèmes de l'Église catholique au début du $19^{\mathrm{e}}$ siècle. Ces différences d'attitude se révèlent dans les relations officielles entre l'évêque Denaut et le coadjuteur Plessis, relations rendues difficiles par la décision de Denaut de rester dans sa paroisse de Longueuil tandis que Plessis est à Québec, mais facilitées par l'amitié entre eux. Avec la mort de Denaut et l'accession de Plessis au siège épiscopal, l'Église passe de la résistance passive à la reconquête du terrain.
Tous droits réservés (C) Les Éditions Historia Ecclesiæ Catholicæ Canadensis Inc., 1984
Ce document est protégé par la loi sur le droit d'auteur. L'utilisation des services d'Érudit (y compris la reproduction) est assujettie à sa politique d'utilisation que vous pouvez consulter en ligne. 


\title{
L'apprivoisement du pouvoir:
}

\section{l'apprentissage épiscopal de Pierre Denaut et de Joseph-Octave Plessis}

\author{
James H. LAMBERT \\ rédacteur historien \\ Dictionnaire biographique du Canada
}

\section{RÉSUMÉ}

Le caractère et la carrière épiscopale respectifs de Pierre Denaut, évêque de Québec de 1797 à 1806, et de son successeur Joseph-Octave Plessis, expliquent en partie leurs façons différentes d'aborder les problèmes de l'Église catholique au début du 19' siècle. Ces différences d'attitude se révèlent dans les relations officielles entre l'évêque Denaut et le coadjuteur Plessis, relations rendues difficiles par la décision de Denaut de rester dans sa paroisse de Longueuil tandis que Plessis est à Québec, mais facilitées par l'amitié entre eux. Avec la mort de Denaut et l'accession de Plessis au siège épiscopal, l'Église passe de la résistance passive à la reconquête du terrain.

Pierre Denaut et Joseph-Octave Plessis: deux évêques de Québec dans le premier quart du $19^{\circ}$ siècle, mais deux styles de gouvernement diocésain fort contrastés. À tel point que la venue de Plessis comme successeur de Denaut à la tête du diocèse de Québec constitue un tournant dans l'histoire de l'Église catholique au Québec.

À quoi attribuer ce contraste? Certainement pas aux origines sociales des deux hommes, car si Denaut, né en 1743 à Montréal, est le $7^{`}$ fils d'un 
maçon prospère, Plessis, de 16 années son cadet, est aussi né à Montréal (en 1759 donc) $77^{\circ}$ enfant d'un tanneur prospère. Puis, Denaut reçoit son éducation dans une école latine dirigée par les sulpiciens, et Plessis fréquente l'école latine que dirige le sulpicien Jean-Baptiste Curatteau. Tous deux poursuivent leurs études au Séminaire de Québec. Ensuite, Denaut est pendant un certain temps secrétaire du presqu'évêque de Montréal, Étienne Montgolfier; Plessis, quant à lui, remplit la fonction de secrétaire des évêques de Québec de 1783 à 1797.

C'est à partir des nominations à leurs cures respectives que Denaut et Plessis montrent des profils de carrière divergents. Denaut exerce son ministère dans des paroisses rurales: Saint-Pierre, à l'île d'Orléans, puis Saint-Joseph, aux Cèdres, et enfin Longueuil. À Longueuil notamment, le peuple, composé de cultivateurs canadiens, est, selon ses propres dires, «bon, docile, religieux». Plessis, par contre, se voit confier la paroisse urbaine de Notre-Dame de Québec où vit une population des plus hétérogène: cultivateurs et commerçants, travailleurs et fonctionnaires, matelots et prostituées, Canadiens et Britanniques, catholiques et protestants. Là, les idées révolutionnaires disputent le terrain à celles de l'Ancien Régime. Là aussi, se trouve le centre politique de la colonie.

À Longueuil, même si sa paroisse offre peu de défis de taille, Denaut impressionne l'évêque Jean-François Hubert, qui le nomme archiprêtre en 1788, puis vicaire général en $1790^{1}$. Denaut s'impose ${ }^{2}$. Toutefois, les vicaires généraux les plus influents du district de Montréal sont successivement Montgolfier et Gabriel-Jean Brassier, tous deux supérieurs du séminaire de Saint-Sulpice.

Influent aussi, le secrétaire Plessis, disponible, énergique, compétent. $\mathrm{M}^{\mathrm{rr}}$ Hubert, qui est souvent malade ou en visite pastorale, ne compte nullement sur son coadjuteur d'avant 1794, Charles-François Bailly de

\footnotetext{
' Sur les origines sociales, l'éducation et les débuts de la carrière de Pierre Denaut, voir sa biographie par Jean Hamelin et Michel Paquin dans le Dictionnaire biographique du Canada (8 vol. parus, Québec, 1966- ), V: 269 (désormais $D B C$, V). Sur Plessis, voir James H. Lambert, «Monseigneur, the catholic bishop; Joseph-Octave Plessis, church, state, and society in Lower Canada; historiography and analysis» (thèse de Ph.D., université Laval, [1981]), 188-214, 217-238.

Lettre de $M^{\mathrm{gr}}$ Hubert à Pierre Denaut. 15 oct. 1792. Archives de l'Archidiocèse de Québec (désormais AAQ), 210A. I: 356. À cette occasion. Hubert félicite Denaut d'avoir établi son autorité dans son district. Toutefois, à unc autre occasion, il lui reproche de ne pas surveiller suffisamment le comportement des prêtres sous sa juridiction (lettre du 16 février 1792. AAQ. 210A. I: 300-301) et. à quelques reprises. il trouve que Denaut ne lui fournit pas suffisamment de détails sur des problèmes à régler (lettres du 21 mars 1791. du 15 octobre 1792 et du 14 octobre 1793. AAQ, 210A. I: 215-216. 355: II: 98-99.)
} 
Messein; il s'en remet plutôt à Plessis et au vicaire général Henri-François Gravé de La Rive. De plus, comme il déteste les relations sociales, il laisse à son secrétaire le soin d'établir et de maintenir les contacts avec l'administration coloniale, de négocier les accords, même si c'est lui qui, en tant qu'évêque, prend les décisions finales. Plessis acquiert donc expérience et confiance.

Humble et peu ambitieux, Denaut n'envie pas le secrétaire. Les deux hommes sont devenus amis. Denaut s'inquiète même pour l'avenir de Plessis, dont l'influence grandissante suscite des plaintes vers 1790; il lui conseille la prudence, mais non d'aller jusqu'à démissionner: «Vous êtes à votre place, Mon Cher, lui écrit-il; vous la devez à la providence; vous la devez à la confiance de votre évêque; vous la devez à vos talents. Quoiqu'en disent vos ennemis, ils le voyent. $\gg^{3}$

En 1794, Hubert, probablement influencé par le gouverneur Lord Dorchester, choisit Denaut comme coadjuteur, à la suite de la mort de Bailly. Mais le nouveau coadjuteur reste à Longueuil, afin, parait-il, d'habituer le gouvernement à voir un évêque dans le district de Montréal et de préparer ainsi le chemin à une division du diocèse. Hubert correspond fréquemment avec Denaut, mais c'est le plus souvent pour l'informer de décisions et d'événements ou pour faire exécuter des tâches administratives. Seule la fondation d'une caisse ecclésiastique - au sujet de laquelle Denaut recommande un projet plus restreint - reflète son influence sur une question de politique. En revanche, l'influence de Plessis, secrétaire expérimenté, se fait beaucoup sentir dans l'administration diocésaine. Au point que lorsque Hubert démissionne en 1797, le choix du coadjuteur pour le nouvel évêque diocésain, Denaut, ne se pose même pas: c'est Plessis.

L'amitié qui lie Denaut et Plessis facilite leurs relations officielles. «J'aime à vous voir vous épanouir avec moi», écrit l'évêque au coadjuteur désigné en novembre 1797. «C'est un délassement bien pardonnable aux tracasseries inséparables de notre état. $»^{4}$

Des tracasseries, Denaut en crée lui-même en décidant de rester à Longueuil, même après être devenu évêque. Au fond il n'a probablement pas envie de quitter sa paroisse tranquille pour le creuset de Québec. S'il pensait exploiter la formule à succès de Hubert, c'était sans compter les différences majeures qui allaient découler du fait que l'évêque ne serait plus

\footnotetext{
${ }^{3}$ Lambert, «Monseigneur, the Catholic bishop», 214-217, 239-246.

${ }^{4}$ Lettre de Denaut à Plessis, 22 novembre 1797. Citée dans Lambert, «Monseigneur, the Catholic bishop», 271.
} 
à Québec. Ainsi Plessis, qui se voit confier l'administration du district de Québec en plus des relations avec l'administration coloniale, se trouve à être plus au fait que l'évêque de l'état général du diocèse'. Le savoir, c'est le pouvoir. Détenant l'information sur un problème quelconque avant l'évêque, Plessis se doit de proposer des solutions. Toutefois, Denaut ne cède pas le gouvernement du diocèse à son coadjuteur; au contraire, par une correspondance régulière, il se tient au courant des affaires et se réserve la décision finale dans les cas d'une importance certaine, particulièrement les nominations de curés. Et il prévient Plessis en juillet 1798: «Quand j’ai terminé une affaire après un mûr examen $[\ldots]$ je m' arrête là, je ne change point. $\aleph^{6}$ Mais l'homme qui est devenu coadjuteur désigné à l'âge de 38 ans ne manque pas de force de caractère non plus. Henri-François Gravé de La Rive écrit à propos des pouvoirs de vicaire général que lui a accordés l'évêque: «J'en use peu parce que $\mathrm{M}^{r}$ Jos. Octave Plessis son coadjuteur est son $1^{\text {er }} \mathrm{G}$.V. comme de raison et c'est une forte tête.»?

En restant à Longueuil Denaut éloigne le pouvoir décisionnel dans l'Église à la fois de l'administration centrale du diocèse et du gouvernement civil. Le courrier Québec-Montréal-Québec prend facilement une semaine à dix jours sans compter le temps de réflexion et de décision nécessaire à Denaut. Et l'évêque a comme principe que «la précipitation, dans les moindres choses, ne [vaut] jamais rien». De plus, à l'occasion il met de côté même les problèmes les plus pressants de l'administration diocésaine pour s'occuper de sa grande paroisse, qui compte au-delà de 1,400 communiants ${ }^{8}$. Les failles dans cet arrangement se révèlent en décembre 1798 après à peine plus d'un an de fonctionnement. Le gouverneur Robert

\footnotetext{
${ }^{5}$ Lettre de Denaut à Plessis, 5 novembre 1798, AAQ, 210A, IV: 12. Révélatrice, cette phrase: «Il parait par votre dernière que nos affaires vont bien $[\ldots] \&$ je remercie la Providence du soin particulier qu'elle prend du Diocèse.»

${ }^{6}$ Lettre de Denaut à Plessis, 24 juillet 1798, AAQ, 210A, II: 363. Les évêques sont en désaccord à quelques reprises sur l'affectation de curés. Dans une lettre du 24 septembre 1804, Denaut affirme que ses derniers arrangements pour la nomination de plusieurs curés est «sans retour». «J'en resterai là, insiste-t-il. Je ne suis point une girouette à tout vent.» AAQ, 210A, IV: 172-73. Cité dans Lambert, «Monseigneur, the Catholic bishop», 272.

? Lettre de Henri-François Gravé de La Rive à son frère, 25 octobre 1797. Archives du Séminaire de Québec, Lettres, S, Projets de lettres et correspondance de M. Gravé en Angleterre, 1790-1801, 6 bis AA. Cité dans Lambert, «Monseigneur, the Catholic bishop», 272.

${ }^{8}$ Denaut lui-même se plaignait des ennuis que lui causaient les postes. Quand Plessis reçoit ses bulles de coadjuteur, il les envoie à Denaut, qui se plaint de leur lenteur à lui parvenir: «Ce n'est pas la première fois que dans des affaires importantes, elles [des lettres] me parviennent trop tard, et qu'elles occasionnent le retardement de deux postes pour les réponses.» Lettre de Denaut à Plessis, 10 octobre 1800, AAQ, 210A, IV: 60. Pour la grandeur de sa paroisse et la tendance de Denaut à s'occuper des affaires paroissiales en priorité, voir Denaut à Hubert, 7 février 1796, et Denaut à Plessis, 3 juin 1805, AAQ, 210A, II: 104; IV: 197.
} 
Prescott se propose de célébrer par une journée d'action de grâces la victoire de Nelson sur la flotte française à la baie d'Aboukir, l'été précédent. Plessis et l'évêque anglican de Québec, Jacob Mountain, accueillent chaleureusement cette idée. Plessis demande donc à Denaut un mandement annonçant une grand-messe. Loin du climat fébrile qui règne dans les cercles du pouvoir à Québec, Denaut écrit un mandement laconique qui annonce la célébration d'un office sobre, le $1^{\text {er }}$ janvier 1799. Entre-temps, de peur que le mandement de Denaut ne lui parvienne pas à temps, pour être imprimé et distribué, Plessis en rédige un, lui aussi, «qui répond à l'enthousiasme du quartier-général», ainsi qu'il en informe l'évêque. Il annonce qu'une grandmesse solennelle sera célébrée le 10 - non pas le $1^{\text {er }}$ - janvier 1799. En l'absence de l'évêque, explique-t-il, «on fait de son mieux».

Les mandements manuscrits se croisent en chemin entre Québec et Montréal. Chaque destinataire est consterné du ton de celui qu'il reçoit. Denaut affirme qu'une grand-messe n'est pas en usage en pareil cas et ne veut pas innover. Plessis souligne que l'avocat général Jonathan Sewell, qui est chargé du programme de la journée, exige «quelque chose d'extraordinaire et de nouveau». De plus, les dates ne correspondent pas. Plessis consulte les deux autres vicaires généraux de la ville de Québec, Gravé et Philippe-Jean-Louis Desjardins. On décide de joindre l'introduction du mandement de Denaut aux ordonnances de celui de Plessis. Puis, ce texte hybride est publié sans plus de consultation avec Denaut. La grand-messe a lieu le 10 janvier".

À quelques autres occasions, l'évêque et son coadjuteur se trouvent en désaccord à propos notamment de nominations de curés et de l'assermentation des catholiques sur la bible protestante dans les cours de justice. (Plessis n'y voit pas d'objection, tandis que Denaut s'y oppose. $)^{10}$ Denaut devient quelque peu agacé par l'indépendance d'esprit et l'entêtement de son coadjuteur. Après la réception des bulles tant attendues de Plessis et sa consécration comme évêque de Canathe in partibus infidelium en janvier 1801, Denaut lui envoie de nouvelles lettres de pouvoirs. Contrairement aux lettres de vicaire général qu'il lui a envoyées en 1797 et qui témoignent d'une confiance spontanée et entière, celles-ci sont teintées de défiance et de suspicion. Toutes les actions et ordonnances antérieures de Plessis sont ratifiées, mais à trois conditions: la première - «qu'il [Plessis] ne puisse rien décider à l'encontre de la teneur de nos mandements déjà publiés ou qui le seront dans le futur»; la deuxième - «qu'il n'entreprenne aucune affaire

\footnotetext{
${ }^{9} \mathrm{~L}$ 'incident est raconté en plus grand détail dans Lambert, «Monseigneur, the Catholic bishop», pp. 273-279.

${ }^{10}$ En ce qui concerne les nominations aux cures, voir la note 6 . Pour la controverse concernant l'assermentation, voir Lambert, «Monseigneur, the Catholic bishop», 726-728.
} 
d'importance, si ce n'est qu'après nous avoir informé de son conseil ou de son dessein»; la troisième - «qu'il soit tenu de nous transmettre dans le plus bref délai toutes et chacune des pièces qu'il aura lui-même préparées ou fait préparer, en rapport avec [ses] fonctions»."

Règle générale, toutefois, les deux évêques maintiennent un bon commerce. Ils se secondent efficacement quand il s'agit de défendre les droits de l'évêque à ériger les paroisses face aux prétentions légales du gouvernement colonial. Denaut informe Plessis des principes à maıntenır; Plessis le conseille sur les moyens à prendre et fait le travail de politicien '2. Puis, en 1802, lorsque le lieutenant-gouverneur sir Robert Shore Milnes proclame une journée d'action de grâces pour célébrer la signature du Traité d'Amiens, Denaut, sans hésitation, annonce par mandement une grandmesse.

À l'instigation de Milnes s'élabore un plan pour augmenter le poids de l'exécutif du gouvernement. Font partie de ce plan les décisions de réduire l'influence qu'exerce le clergé catholique sur le plan social et, par consćquent, politique, et de la canaliser au profit de l'État. Le statut légal de l'Église catholique - institution non reconnue par les lois britanniques est contesté par Sewell dans les cours de justice de la province ${ }^{13}$. En février 1805, espérant profiter de l'éloignement géographique de Denaut et Plessis, Milnes se rend à Longueuil offrir à Denaut la reconnaissance civile de l'évêque de Québec en retour de certains pouvoirs que lui conteste l'autorité civile. Mais Denaut se retranche derrière un mur de prudence. En avril, Milnes tente sa chance auprès de Plessis. La discussion s'enlise dans des problèmes d'ordre juridique, et Milnes délègue à Sewell la mission de mener l'affaire à terme. Plessis demande à Denaut l'autorisation de continuer à rencontrer Sewell. Toutefois, il n'attend pas les trois semaines que Denaut met à répondre; il rencontre Sewell au moins une fois entre-temps. D'autres réunions suivent, mais les discussions achoppent principalement sur les droits de l'évêque, contestés par le gouvernement, de nommer aux cures et

\footnotetext{
"J'ai à remercier l'abbé Armand Gagné, des Archives de l'Archevêché de Québec pour ce renseignement. Frappé par la différence de ton entre les lettres de vicaire général que Denaut a données à Plessis en 1797 et les lettres de pouvoirs de 1801, l'abbé Gagné a attiré mon attention là-dessus et m'a fourni une traduction française des conditions contenues dans les lettres de 1801. La nature de ces conditions laisse penser que Denaut avait en tête encore à cette date l'incident du mandement annonçant l'office d'action de grâces en 1799.

12 Voir Lambert, «Monseigneur, the Catholic bishop, 291-297, 334-336.

${ }^{13}$ Sur Sewell, voir Jean-Pierre Wallot, «Sewell et son projet d'asservir le clergé canadien (1801)», Revue d' histoire de l' Amérique française, 16 (mars 1963), 549-566, révisé et publié dans Jean-Pierre Wallot, Un Québec qui bougeait: trame socio-politique du Québec au tournant du XIXe siècle (Québec, 1973), 169-182, et Lambert, «Monseigneur, the Catholic bishop», 328-334.
} 
de créer des paroisses. Plessis envoie à Denaut un compte rendu de chaque rencontre.

Vers la fin de mai 1805, les discussions sont dans l'impasse. De peur de voir échapper la reconnaissance civile de l'évêque de Québec, Plessis exhorte Denaut à la demander quand même au roi par pétition. Il envoie à Longueuil un brouillon d'une pétition à Milnes, d'une autre au roi et de lettres patentes nommant Denaut évêque de Québec et détaillant ses fonctions épiscopales: c'est là, dans la pensée de Plessis, l'instrument qui garantit le statut juridique de l'évêque. Pour obtenir la reconnaissance civile, il recommande de faire certaines concessions au gouvernement, telles la nomination par l'État avec la participation de Rome, du coadjuteur. un droit de veto pour l'État sur la nomination et la destitution des curés, et une voix accordée à l'État dans l'érection des paroisses. Denaut trouve le prix de la reconnaissance civile élevé. "Ôtez en effet à cet évêque la nomination aux cures et fixez les curés; que lui reste-t-il à faire? Quel bien pourra-t-il procurer dans son diocèse? Quel mal pourra-t-il arrêter?» Le peuple croirait à une trahison. Denaut préfère le statu quo. Il rejette les brouillons de Plessis. Il veut prendre tout le temps nécessaire pour réfléchir.

Mais Plessis est pressé: Milnes retourne bientôt en Angleterre; l'ouverture politique qu'il représente se fermera. Frustré, le coadjuteur voit à tort dans les hésitations de Denaut la marque du vicaire général Jean-HenriAuguste Roux, supérieur des sulpiciens. Il écrit à Roux:

Ne vaut-il pas mieux rester comme nous sommes, dites-vous! Mais comment sommes-nous? Sans état fixe, sans autorisation, soumis aux dispositions des instructions du roi qui donne tout uniment au gouverneur la provision (collation) de toutes les cures en l'autorisant à ne déplacer les curés que quand ils seront convaincus de félonie. Avouons que l'état de l'évêque tel que suggéré dans mon plan de lettres patentes, quoique dépendant et gêné, serait bien préférable à celui d'à présent et il vaudrait mieux céder quelque chose que de tout risquer. Après Dieu, la religion catholique dans ce pays n'offre de protection à ses ministres que dans le gouvernement. Les fidèles les plus zélés sont dans les basses classes du peuple. La haute classe de catholiques, conseillers, juges, avocats, marchands de quelque crédit, n'est point en général amie du clergé [...]. Le gouverneur et l'avocat général savent très bien que nous avons en horreur l'anglicanisme et principalement la suprématie du Roi qui en fait partie. Je le leur ai déclaré à pleine bouche et plusieurs fois, en ajoutant même que l'évêque ne pourroit en conséquence accepter une commission qui en impliquerait la plus petite ombre. Ils ne voient donc dans nos places ecclésiastiques que le temporel [...]. Le spirituel est à nous. ${ }^{14}$

\footnotetext{
${ }^{14}$ Lettre de Plessis à Roux, 3 juin 1805, AAQ, 20A, III: 139.
} 
Trois jours plus tard, Plessis écrit à Denaut qu “il partage ses appréhensions, «mais non dans toute leur étendue».

Il faut avouer, Monseigneur. que nous sommes dans un moment de crise pour la religion, insiste-t-il. Tous les jours l'église du pays perd de cette autorité extérieure dont elle a besoin pour soutenir le spirituel et pour faire l'oeuvre de Dieu. Les droits qu'exerce ici Votre Grandeur sont contestés non seulement par les avocats protestans mais par les catholiques [...] dans les cours de justice. On met en question la validité de l'institution des curés faite sans la participation dụ goı̣vernement Bientôt ils ne seront plus reçus à poursuivre le payement de leurs dîmes."

Denaut semonce Plessis pour ses observations à Roux: «assurément il ne s'y attendait pas; moi encore moins», écrit-il. Un deuxième brouillon de pétitions, rédigé également par Plessis, est jugé plus satisfaisant, mais rejeté lui aussi. Denaut en fait un troisième. Plessis gagne donc sur le principe de demander au roi la reconnaissance civile; mais sans les lettres patentes définissant les droits civils de l'évêque, la victoire n'est qu'apparente. De plus, la pétition de Denaut laisse au roi la décision quant aux droits à accorder à l'évêque. Content, Milnes part pour l'Angleterre le 5 août 1805 , la pétition au roi dans ses bagages. ${ }^{16}$

La fin des tractations au sujet de la requête permet un rétablissement des bonnes relations entre les évêques. Le 6 janvier 1806, Denaut invite le coadjuteur à venir se reposer à Montréal. «Rien ne peut être plus agréable que le plaisir de vous voir», rassure-t-il. Deux semaines plus tard, une lettre de Roux informe Plessis de «la prompte et déplorable mort de Monseigneur notre Évêque». ${ }^{17}$

Plessis s'en va enterrer Denaut dans l'église de la paroisse qu'il ne s'était jamais résigné à quitter. De retour à Québec, en moins de deux semaines, il se fait assermenter comme évêque de Québec et fait accepter un coadjuteur en la personne de Bernard-Claude Panet. Compte tenu de la vive opposition à ces deux actions manifestée par les deux fonctionnaires les plus influents de la colonie, Sewell et le secrétaire civil et greffier du Conseil exécutif Herman Witsius Ryland, il s'agit de coups d'éclats.

À son tour évêque de Québec, Plessis affiche, au départ, la même prudence que Denaut. Le gouverneur sir James Henry Craig a un comporte-

\footnotetext{
15 Lettre de Plessis à Denaut, 6 juin 1805, AAQ, 20A, III: 141.

16 Sur toute cette affaire, voir Lucien Lemieux. L'Établissement de la première province ecclésiastique au Canada 1783-1844 (Montréal et Paris. Fides. 1968). 51-56. et Lambert. «Monseigneur, the Catholic bishop». 337-352.

${ }^{17}$ Lettre de Denaut à Plessis, 6 janvier 1806; lettre de Roux à Plessis, 10 janvier 1806, AAQ, 210A, VI: 691; VII: 748. Sur les relations entre Plessis et Denaut après les négociations éprouvantes du printemps et de l'été de 1805 , voir Lambert, «Monseigneur, the Catholic bishop», 356-357.
} 
ment qui rappelle celui de Prescott et de Milnes. Par ses actions, Plessis reconnaît que Denaut avait raison de ne pas faire de concessions préalablement à la reconnaissance civile. Mais si la prudence de Denaut a peut-être sauvé la situation en 1806, c'est le dynamisme de Plessis qui, à la longue, changera l'histoire de l'Église. Quoique à certains moments compatissant, chaleureux, magnanime, Denaut était au fond solitaire, distant, peu loquace, même frondeur à l'occasion; il n'était point l'homme à nouer les relations humaines qui auraient permis de tâter le pouls de l'Église et d'inspirer le clergé. C'était un évêque pastoral, comme en témoignent son zèle pour sa paroisse et son empressement à faire des visites pastorales jusque dans le Haut-Canada et les Maritimes (souci que partage son coadjuteur, d'ailleurs). Mais on cherche en vain chez Denaut une volonté de s'attaquer aux problèmes structurels et conjoncturels du diocèse; il trouve prématurés les efforts en vue de le diviser malgré son immensité, ainsi que pour obtenir une reconnaissance civile du clergé; il manifeste peu d'enthousiasme pour l'importation des prêtres français, mais fait peu pour encourager le recrutement de Canadiens en dépit d'une grave pénurie de prêtres; il trouve imprudent un projet de caisse ecclésiastique conçu non seulement pour venir en aide au clergé mais aussi pour financer des projets diocésains à une époque où les évêques manquent de ressources financières. Formé dans des paroisses rurales paisibles, Denaut ne voit à l'extérieur de ces milieux que dangers à éviter; tandis que, aguerri dans ce creuset qu'est Québec, Plessis ne voit qu'obstacles à surmonter. De Denaut à Plessis, on passe de la résistance passive au début de la reconquête du terrain, surtout à partir de 1811 , dans un contexte politique plus propice. Bien placé à Québec pour être adéquatement renseigné et pour agir rapidement, doté d'un dynamisme et d'un acharnement nés de la conviction qu'il gagnera à la fin, Plessis s'attaque aux problèmes de l'Église. Si ses gains sont souvent des demisuccès - reconnaissance civile de l'évêque limitée à sa propre personne, division boiteuse du diocèse, lenteur frustrante dans l'accroissement du recrutement local du clergé, loi des écoles de fabriques abrogée après sa mort, révision du petit catéchisme mais abandon de celle du grand, etc. ils n'en constituent pas moins un départ vers quelque chose de mieux. Plessis imprime à l'Église et au clergé une confiance, une conviction, et un sens de direction qu'ils n'avaient pas connus depuis la Conquête et probablement depuis bien avant. Il donne à l'Église catholique un momentum que d'autres maintiendront et qui la portera à travers les tumultueuses années 1830 et l'installera à la tête de la société canadienne au lendemain de la débâcle de la bourgeoisie nationaliste en 1837-1838. 